\title{
Monocytic CCR2+ Myeloid Derived Suppressor Cells Promote Immune Escape By Limiting Activated CD8 T Cell Infiltration Into The Tumor Microenvironment
}

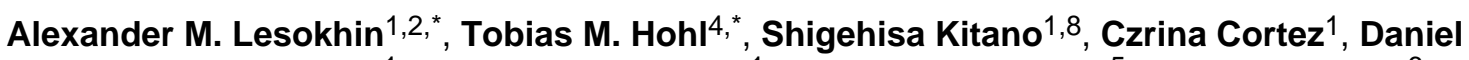 \\ Hirschhorn-Cymerman ${ }^{1}$, Francesca Avogadri ${ }^{1}$, Gabrielle A. Rizzuto ${ }^{5}$, John J. Lazarus ${ }^{6}$, \\ Eric G. Pamer ${ }^{1,2,3}$, Alan N. Houghton ${ }^{1,2,3}$, Taha Merghoub ${ }^{1, \dagger}$, and Jedd D. Wolchok ${ }^{1,2,7,8, \dagger}$ \\ ${ }^{1}$ Memorial Sloan-Kettering Cancer Center, New York, New York \\ ${ }^{2}$ Weill Medical College, Cornell University, New York, New York \\ ${ }^{3}$ Graduate School of Medical Sciences of Cornell University, New York, New York \\ ${ }^{4}$ Vaccine and Infectious Disease Division, Fred Hutchinson Cancer Research Center, Seattle, \\ Washington \\ 5University of California, San Francisco Medical Center, San Francisco, California \\ ${ }^{6}$ University of Toledo College of Medicine, Toledo, Ohio \\ ${ }^{7}$ Ludwig Institute for Cancer Research-NY Branch \\ ${ }^{8}$ Ludwig Center for Cancer Immunotherapy at Memorial Sloan-Kettering Cancer Center
}

\begin{abstract}
Myeloid derived suppressor cells (MDSC) are a heterogeneous population of cells that accumulate during tumor formation, facilitate immune escape and enable tumor progression. MDSC are important contributors to the development of an immunosuppressive tumor microenvironment that blocks the action of cytotoxic anti-tumor $\mathrm{T}$ effector cells. Heterogeneity in these cells poses a significant barrier to studying the in vivo contributions of individual MDSC subtypes. Herein, we demonstrate that granulocyte-macrophage colony stimulating factor (GM-CSF), a cytokine critical for the numeric and functional development of MDSC populations, promotes expansion of a monocyte-derived MDSC population characterized by expression of CD11b and the chemokine receptor CCR2. Utilizing a toxin mediated ablation strategy to target CCR2-expressing cells, we show that these monocytic MDSCs regulate entry of activated CD8 T cells into the tumor site, thereby limiting the efficacy of immunotherapy. Our results argue that therapeutic targeting of monocytic MDSCs would enhance outcomes in immunotherapy.
\end{abstract}

Corresponding Author: Jedd D. Wolchok MD, PhD, Memorial Sloan-Kettering Cancer Center, 1275 York Avenue, New York NY

10065, wolchokj@mskcc.org, phone: 646-888-2395, fax: 646-422-0453.

*These authors contributed equally

Co-senior authors

Authorship

A.M.L. designed and performed research, analyzed data, and wrote the paper; T.H. contributed vital reagents, designed research, analyzed data, and contributed to writing the paper. S.K., C.C., D.H.C., G.A.R. performed research and contributed to writing the paper; F.A. and J.L performed experiments; E.G.P. contributed vital reagents and contributed to writing the paper; A.N.H. designed experiments, analyzed data and contributed to writing the paper: T.M. and J.D.W. designed research, analyzed data, and wrote the paper.

The authors have no conflicting interests. 


\section{Keywords}

Granulocyte Macrophage Colony Stimulating Factor; Myeloid Derived Suppressor Cells; CC

Chemokine Receptor 2; Melanoma

\section{Introduction}

A requirement for immunotherapy approaches against cancer is the expansion and entry of tumor antigen specific $\mathrm{T}$ cells into the tumor microenvironment. However, established tumors develop an anti-inflammatory stroma and recruit immunosuppressive cells to the tumor site, both of which prevent immune eradication even when tumor antigen-specific $\mathrm{T}$ cells are detectable in the periphery ${ }^{1,2}$. Myeloid derived suppressor cells (MDSC) ${ }^{3}$, a heterogenous population of myeloid cells, represent an immunosuppressive cell population that has been noted in many pre-clinical tumor models and in humans with cancer.

More than 20 factors have been described in MDSC expansion in the context of both murine and human tumors ${ }^{4}$. Among these, GM-CSF an immune stimulatory cytokine in some situations appears to also be critical for MDSC expansion and function. Recent negative results of randomized clinical trials utilizing GM-CSF as an adjuvant underscore the importance of further characterizing the role of GM-CSF expanded MDSC in immune based therapies $^{5-7}$.

MDSC consist of mononuclear, polymorphonuclear (PMN), and immature myeloid cells with differential ability to suppress T cell function via the coordinated function of iNOS and arginase ${ }^{8}$. MDSC heterogeneity complicates the study of this population because of differing interpretations on how MDSC constituents fit within myeloid cell developmental lineages and contribute to tumor induced immune suppression 9,10 . In mice, MDSCs express the myeloid antigens CD11b and Gr-1 (which stains both Ly6C and Ly6G) found on monocytic and neutrophilic cells. Other markers for these cells include F4/80, CD80, CD115, and IL4-R $\alpha^{11-13}$. More recently, Gr-1 or Ly6G with Ly6C expression have been found to identify a PMN population (CD11b+, Ly6G+/Ly6C int or Gr-1 high) and a mononuclear population (CD11b+, Ly6G-/Ly6C high or Gr-1 int/low) ${ }^{14-16}$ that expand to varying degrees and have differing suppressive capability in different tumor models, likely due to variations in tumor-secreted factors that mediate their expansion and/or activation. How these different MDSC subsets function in the context of distinct tumor secreted factors remains poorly understood. Furthermore, tools to specifically track and deplete a single subset of MDSC in vivo have not been developed.

In this study we find that GM-CSF is critical for the expansion and function of monocytic, but not granulocytic MDSC. We define monocytic MDSC by their co-expression of CD11b and CCR2. CCR2+MDSC are present in multiple tumor models and in patients with melanoma. Importantly, depletion of this cellular subset using recently described transgenic mice that enable the tracking and inducible depletion of CCR2+ cells defines a novel role for these cells in limiting antigen specific $\mathrm{T}$ cell entry into the tumor and thereby promoting tumor progression.

\section{Methods}

Mice

Mice were maintained and bred in a pathogen-free vivarium and all procedures were performed in accordance with institutional guidelines at Memorial-Sloan Kettering Cancer Center (MSKCC) under an IUCAC approved protocol. C57BL/6J mice (females, 6-8 wk 
old) and CSF2R $\beta \mathrm{c}-1-$ mice were obtained from The Jackson Laboratory (Bar Harbor, ME). CCR2 knockout mice were obtained from Eric Pamer. CCR2-GFP reporter and CCR2-DTRCFP depleter mice were generated by Tobias Hohl, as previously described in ${ }^{17}$. Thy $1.1^{+}$ pmel-1 TCR transgenic mice have been previously reported ${ }^{18}$.

\section{Cell lines and tumor challenge}

B16F10 (referred to as B16), a mouse melanoma cell line of C57BL/6J originally obtained from I. Fidler (M.D. Anderson Cancer Center, Houston, TX) has been passaged in vivo 4 times to obtain reproducible tumor growth. B16-GM was generated by transduction of B16F10 with the GM-CSF gene as previously described ${ }^{19}$. EL4, EG7, and TRAMP-C2 cell lines were obtained from the American Type Culture Collection (ATCC, Manassas, VA). B16, B16-GM, and TRAMP-C2 cell lines were cultured as described ${ }^{20,21}$. EL4 and EG7 were cultured in RPMI supplemented with 7.5\% heat-inactivated FCS (ATCC), $200 \mathrm{U} / \mathrm{ml}$ penicillin, and $50 \mathrm{mg} / \mathrm{ml}$ streptomycin (Invitrogen, Carlsbad, CA). For tumor challenge experiments, $1.25 \times 10^{5}$ B16 or B16-GM cells, $5 \times 10^{5}$ EL4 or EG-7 cells, and $1 \times 10^{6}$ TRAMP-C 2 cells are injected intradermally into the shaved right flank of a mouse. Tumor growth was monitored every 2-3 days. Mice were sacrificed if tumors exceeded $1 \mathrm{~cm}$ in diameter or became ulcerated, or if mice showed signs of discomfort. For ex vivo analysis of immune infiltration into tumors or purification of tumor infiltrating MDSC subsets mice were injected subcutaneously with the indicated numbers of tumor cells reconstituted in growth factor reduced Matrigel $^{\mathrm{TM}}$ (BD Biosciences, Franklin Lakes, NJ) collagen matrix.

\section{Antibodies and flow cytometry}

Mouse tumor samples were finely chopped and treated with collagenase $2 \mathrm{mg} / \mathrm{ml}$ (Roche, Indianapolis, IN) and DNase $1 \mathrm{mg} / \mathrm{ml}$ (Sigma, St. Louis, MO) in PBS at $37^{\circ} \mathrm{C}$ for 45 minutes. All tissues were mashed through $8 \mu \mathrm{m}$ filter to generate single cell suspensions in RPMI supplemented with 7.5\% fetal calf serum. After RBC lysis (ACK lysis buffer, Lonza, Basel, Switzerland) when required, samples were washed in FACs buffer (PBS/1\%BSA/ 2mM EDTA), incubated in Fc block (CD16/32, clone 2.4G, BD Biosciences, Franklin Lakes, NJ) and stained for 25 minutes at $4^{\circ} \mathrm{C}$ with the relevant Abs. Abs used for analysis of tumor infiltrate were Ly6C FITC, CD4 PerCP, CD8 PE-Cy7, CD11b APC-Cy7 from BD Biosciences (Franklin Lakes, NJ) and FoxP3 APC (intracellular) from eBioscience (San Diego, CA). Additional Abs used for T cell adoptive transfer experiments were Ly6G PE, Thy 1.1 PerCP, CD11b Percp-Cy5.5, CD45 APC-Cy7 from BD Biosciences (Franklin Lakes, NJ) and Ly6C AF647 from Abd Serotec (Raleigh, NC). Live/Dead fixable aqua dead stain or 4',6-diamidino-2-phenylindole (Invitrogen, Carlsbad, CA) was added to cells as appropriate to assess viability prior to acquisition on an LSR2 flow cytometer. For human samples $5 \times 10^{5}$ PBMCs from melanoma patients or healthy donors were washed with $2 \mathrm{ml}$ FACS buffer (phosphate-buffered saline containing $2 \%$ bovine serum albumin and $0.05 \mu \mathrm{M}$ EDTA). The following antibodies were then added for $20 \mathrm{~min}$ at $4^{\circ} \mathrm{C}$ : Lineage (CD3/CD16/ CD19/CD20/CD56) cocktail FITC (special-ordered BD Pharmingen), CD14-PerCP Cy5.5, CD11b-APC Cy7, CD33-PE-Cy7 (BD Pharmingen), HLA-DR-ECD (Beckman Coulter), IL-4R $\alpha$-PE (R\&D Systems, Minneapolis, MN) and CD192 (CCR2)-Alexa Flour 647 (Biolegend, San Diego, CA). Isotype controls included the appropriate fluorochrome conjugated mouse $\operatorname{IgG}_{1}, \operatorname{IgG}_{\mathrm{k}}, \operatorname{IgG}_{2 \mathrm{a}}$, or $\operatorname{IgG}_{2 \mathrm{~b}}$ (BD Pharmingen, Beckman Coulter, R\&D Systems, or Biolegend). The stained cells were detected using a CyAn flow cytometer. All analysis was performed using FlowJo software (Treestar, Ashland, OR).

\section{Purification of MDSC}

Mouse tumor-infiltrating or lymphoid tissue CD11b+ cells were obtained from single cell suspensions generated as in the previous section. Tumor cells were subsequently separated from debris over a Percoll@ (Sigma Aldrich, St. Louis, MO) gradient. B cells were depleted 
from splenocytes using CD19 microbeads and LD columns according to the manufacturer's protocol (Miltenyi Biotec, Bergisch Gladbach, Germany) to enrich myeloid fractions. Cells were stained with CD11b PerCP-Cy5.5, CD45 APC-Cy7 and DAPI for flow sorting on a Cytomation MoFlo or BD FACS-Diva Cell Sorters. Purity of FACs sorted populations was 90-95\%.

For human samples thawed PBMCs were resuspended in $0.5 \%$ human serum PBS at $5 \times 10^{6}$ cells $/ \mathrm{ml}$ and incubated with carboxyfluorescein diacetate succinimidyl ester (CFSE; Invitrogen, Carlsbad, CA) at a final concentration of $0.5 \mu \mathrm{M}$ for $5 \mathrm{~min}$ at room temperature. Cells were washed three times in $0.5 \%$ Human serum PBS. $3 \times 10^{6}$ PBMCs were stained with $10 \mu \mathrm{L}$ of anti human CCR2-PE antibody for $15 \mathrm{~min}$ at $4^{\circ} \mathrm{C}$ and washed in $0.5 \% \mathrm{FBS}$ PBS twice. The cells were then depleted of CCR2-PE ${ }^{+}$cells with anti PE MicroBeads (Miltenyi Biotec) according to the instructions supplied by the manufacturer. These were used as effectors. CCR2+ cells were also flushed from the columns for use as suppressors where indicated. Human PBMC were collected using a Memorial Hospital Institutional Review Board-approved biospecimen protocol (MH \# 00-144) for study of correlative immunologic markers in patients with melanoma.

\section{T cell preparations}

Spleen and lymph nodes were passed through an $8 \mu \mathrm{M}$ filter to generate a single cell suspension. These were subsequently subjected to RBC lysis and magnetically purified using anti-CD8 (Ly-2) microbeads according to the manufacturer's protocol (Miltenyi Biotec, Bergisch Gladbach, Germany). Purity was assessed by flow cytometry and greater than $90 \%$. Cells were labeled with $1 \mathrm{mM}$ or $10 \mathrm{mM}$ carboxyfluorescein diacetate succinimidyl ester (CFSE; Invitrogen, Carlsbad, CA) for in vitro or in vivo studies, respectively. Activated CD8 T cells were generated by culturing splenocytes with soluble $\alpha$ $\mathrm{CD} 3,1 \mu \mathrm{g} / \mathrm{ml}(145-2 \mathrm{C} 11)$ and $\alpha-\mathrm{CD} 28,2 \mu \mathrm{g} / \mathrm{ml}(37 \mathrm{~N})$ for 72 hours. Recombinant human IL-2, 30 units/ml (Chiron, Emeryville, California) was added for the final 24 hours of culture. CD8+ cells were subsequently positively selected with anti-CD8 microbeads prior to injection via tail vein.

\section{Suppression Assay}

$1 \times 10^{5}$ CFSE-labeled splenocytes or CD8+T cells were plated in complete media (RPMI 1640, 10\%FCS, 200 units/ml penicillin, $50 \mathrm{mg} / \mathrm{ml}$ streptomycin) supplemented with $2 \mathrm{mM}$ L-glutamine and $0.05 \mathrm{mM} \beta$-mercaptoethanol onto round bottom 96-well plates coated with $1 \mu \mathrm{g} / \mathrm{ml} \alpha$-CD3 and $5 \mu \mathrm{g} / \mathrm{ml} \alpha$-CD28. MDSC were added in 1:1 ratios unless otherwise indicated. To assess contact dependence, MDSC were placed into transwell inserts with 0.4 $\mu \mathrm{M}$ polycarbonate membranes (Corning ${ }^{\circledR}$ HTS Transwell®, Corning, NY).

For human cells, $2 \times 10^{5}$ CFSE-labeled CCR2 ${ }^{-}$PBMCs with or without CCR2 ${ }^{+}$cells were cultured in 96-well flat-bottom $\alpha$-CD3-specific Ab coated plates (OKT3, $100 \mathrm{mcl}$ at $0.5 \mu \mathrm{g} /$ $\mathrm{mL}$ for 2 hours at $37^{\circ} \mathrm{C}$ ) in RPMI 1640 medium supplemented with 10\% FBS and IL-2 (10 IU/mL; Roche, Mannheim, Germany). After 5 days, cells were harvested, stained with CD3PECy7, CD4-ECD, CD8-APCCy7 (BD Pharmingen) and CFSE signal of gated CD8 ${ }^{+} \mathrm{T}$ cells $\left(\mathrm{CD}^{+} \mathrm{CD}^{-}\right)$was measured by flow cytometry.

\section{Cytokine Measurements}

Mouse blood was obtained by retro-orbital puncture when tumors were $0.5-1 \mathrm{~cm}$ in diameter. Blood was allowed to clot for 30 minutes on ice and centrifuged at 3600 RPM for 5 minutes and serum was aspirated. Tumors were weighed, placed in $500 \mathrm{mcl}$ PBS, and treated in a bead homogenizer for 5 minutes. Debris was removed by 2 rounds of centrifugation at 14,000 RPM to obtain cytokines in solution. Cytokine levels were 
measured using FlowCytomix beads (eBioscience, San Diego, CA) according to the manufacturer's instructions. Samples were acquired on an LSRII flow cytometer and analyzed using FlowCytomixPro software.

\section{Statistics}

All values shown in graphs represent the mean \pm SEM. Statistical differences between different groups were determined by a two-tailed a Student's T test. $\mathrm{p}<0.05$ was considered statistically significant. Correction for multiple comparisons was not performed due to the hypothesis generating nature of these studies. Differences between tumor growth curves were assessed using ANOVA analysis. All graphs and statistical calculations were performed using Prizm software (Graphpad Software, La Jolla, CA).

\section{Results \\ Chronic GM-CSF promotes tumor growth}

In prior studies we observed that live B16 murine melanoma cells engineered to secrete GM-CSF promote concomitant immunity in vivo ${ }^{1,22,23}$. In this model, a primary tumor that secretes GM-CSF is capable of protecting against a second tumor challenge. This effect is CD8+ T cell dependent. However, we found it surprising that the primary GM-CSF secreting B16 tumors (B16-GM) continue to grow despite the presence of protective CD8+ $\mathrm{T}$ cell immunity. To study this further, we assessed the effects of GM-CSF on tumor growth kinetics by challenging C57B16/J mice with B16 or B16-GM tumors. We observed that B16GM led to a more rapid tumor growth in vivo (fig 1A), suggesting that the sum effect of chronic GM-CSF exposure is to promote tumor growth. We found both cell lines had similar growth kinetics in vitro with B16 having a slightly more rapid doubling time than the GMCSF secreting counterpart (fig 1B) making it unlikely that GM-CSF is exerting a direct proliferative effect on the tumor cells. Furthermore, B16 and B16-GM growth differences were abrogated in CSF2 $\beta \mathrm{c}$ receptor knockout mice, which lack the common beta subunit shared by GM-CSF, IL-3, and IL-5 (fig 1C). These data suggest chronic GM-CSF exposure promotes tumor growth indirectly via effects on host cells. Interestingly, both tumor cell lines grew at a slightly increased rate in CSF2 $\beta \mathrm{c}$ knockout mice suggesting a global role for this shared receptor in controlling tumor outgrowth (data not shown).

\section{Tumor derived GM-CSF recruits a cellular infiltrate dominated by myeloid derived suppressor cells (MDSC)}

Since indirect effects on host cells seemed to explain the observed tumor grow kinetics, we examined the effects of chronic GM-CSF exposure on tumor infiltrating immune cell subsets in B16 and B16-GM tumors. B16-GM tumor growth was noted to increase the number of myeloid cells both at the tumor site and in the spleen (fig $2 \mathrm{~A} / \mathrm{B}$ ) in mice bearing B16-GM tumors, while an increase in the absolute number of regulatory T cells (Tregs) was only observed in the spleen (fig 2A). Significantly diminished CD8+ T cell infiltration was noted at the tumor site with mildly diminished numbers seen systemically (fig $2 \mathrm{~A}$ ). To test the prediction that the myeloid cells infiltrating the B16-GM tumor site were myeloid derived suppressor cells, we performed $\mathrm{T}$ cell suppression assays comparing equivalent numbers of CD11b cells purified by flow cytometry from B16 or B16GM tumors for CD8+ $\mathrm{T}$ cell suppressive capability. This showed that $\mathrm{CD} 11 \mathrm{~b}+$ cells isolated from the B16-GM tumor site were MDSC (fig 2C). In contrast, CD11b+ cells isolated from the spleens of B16GM bearing animals did not suppress CD8+ T cell proliferation, suggesting that MDSC at the tumor site were a suppressive subset of $\mathrm{CD} 11 \mathrm{~b}+$ cells present in the tumor microenvironment. Importantly, CD11b+ cells purified from the B16 tumor or from the spleen of B16 tumor-bearing mice were incapable of suppressing CD8+ T cell proliferation. 
In sum, these results show that B16-GM recruits an immune-inhibitory cellular infiltrate characterized by a CD11b+ MDSC population and a relative paucity of CD8+ T cells.

\section{GM-CSF expands monocytic CCR2+ MDSC}

To investigate if GM-CSF expanded MDSC are granulocytic or monocytic cells we focused on CCR2 expression, as this chemokine is required for monocyte trafficking from the bone marrow and is not expressed on granulocytes ${ }^{24}$. Furthermore, monocytic MDSC have been shown to depend on CCR2-mediated signals for tumor trafficking ${ }^{25}$. CCR2 specific antibodies have been reported, but are not commercially available ${ }^{26}$. Using a CCR2-GFP reporter mouse strain, we found that both CCR2+CD11b+ and CCR2-CD11b+ myeloid cells are systemically expanded by GM-CSF with a preferential accumulation of CCR2+CD11b+ cells at the tumor site(fig $3 \mathrm{~A}$ ). As expected, sorting cells based on CCR2 expression concentrates a monocytic population (fig $3 \mathrm{~B}$ ). We next tested which population within the MDSC compartment contained suppressive function. We found that CCR2+CD11b+ myeloid cells purified from the tumor or spleen of mice with B16-GM tumors (but not B16 tumors) contained the $\mathrm{CD} 11 \mathrm{~b}+$ cell fraction capable of suppressing $\mathrm{T}$ cell proliferation; whereas CCR2-CD11b+ cells from either site or tumor model did not lead to significant $T$ cell suppression (fig 3C and not shown) suggesting that CCR2- cells do not contain a dominant MDSC population. Interestingly, CCR2+CD11b+ cells isolated from mice growing EL-4 thymoma, Eg7(its ovalbumin expressing counterpart) and TRAMP-C2 prostate tumors had similar suppressive function (fig S1), but GM-CSF was not detected in the sera or tumor homogenates in these tumor model systems (fig S2). Taken together, these data not only demonstrate that a monocytic CCR2+CD11b+ myeloid cell is the relevant MDSC subset that is expanded, activated and recruited to the tumor site by GM-CSF action, but also that CCR2+MDSC are expanded by other tumor types suggesting that CCR2 expression can be applied more broadly to characterize monocytic MDSC.

\section{CCR2+MDSC inhibition of T cell proliferation is both contact dependent and requires the function of Arginase and iNOS}

MDSC are thought to exert their T cell suppression through release of reactive oxygen species (ROS) and the production of peroxynitrites through the concerted action of arginase and iNOS ${ }^{4}$. In addition, a role for contact-dependent MDSC function has also been proposed ${ }^{27}$. Recent studies have suggested that there is a preferential dependence on ROS versus NO production in the suppressive function of granulocytic versus monocytic MDSC, respectively ${ }^{15}$. Using available inhibitors and transwell assays we tested whether CCR2+MDSC suppressive function depended on one, some, or all of these mechanisms. We found that CCR2+CD11b+ cells inhibited $\mathrm{T}$ cell proliferation in a contact dependent fashion (fig 4A). We also found that both arginase and iNOS (fig 4B) were active in T cell suppression. In the inhibitor experiments we normalized for the level of stimulation induced by $\alpha-C D 3 / \alpha-C D 28$ antibody across 3 separate assays to reflect differences between the maximum to minimum proliferation seen in each experiment. The $1^{\text {st }}$ column in figure $4 \mathrm{~B}$ shows proliferation with MDSC in the absence of inhibitor. Either inhibitor alone improves proliferation by approximately $50 \%$ while both inhibitors synergize to further increase proliferation suggesting both iNOS and Arginase activity in mediating suppressive function. Inhibitors of indoleamine dioxygenase (DL 1-MT) and ROS (catalase) did not restore T cell proliferation (data not shown). These findings are consistent with the MDSC definition and suggest that GM-CSF is sufficient for the expansion of monocytic MDSC with the full complement of suppressive mechanisms predicted for this MDSC constituent.

\section{MDSC suppressive function is CCR2 independent}

The observations that CCR2 reporter expression defines an MDSC subset led us to evaluate the role of this receptor in MDSC function. CCR2 is critically important for monocyte 
egress from the bone marrow in the setting of acute inflammatory events such as infection and a role for this molecule in myeloid cell trafficking to tumors has also been proposed $^{24,25}$. Therefore, we crossed the CCR2 reporter mice (in which the CCR2 locus is disrupted by the GFP gene) onto a CCR2-/- background to identify MDSC that lacked functional CCR2. We found a 50\% reduction in the number of GFP+MDSC at the tumor site with similar reductions seen in the blood and spleen (fig 5A, 5B). A reciprocal increase of GFP+MDSC in the bone marrow was seen. These finding are consistent with results from models of infection and suggest against additional CCR2 dependence for MDSC trafficking from the vasculature to the tumor site. Suppression assays with CCR2 reporter+ MDSC purified from B16-GM tumor bearing animals from both wt and CCR2-/- mice revealed equivalent suppressive capability (Fig 5C) demonstrating that on a per cell basis signaling through CCR2 was not required for MDSC activity.

\section{CCR2+ cells inhibit the therapeutic efficacy of adoptive T cell therapy}

To evaluate the effects of CCR2+ MDSC in vivo we utilized a mouse strain engineered with a transgene that expresses the diphtheria toxin receptor under control of the CCR2 promoter (CCR2 depleter mice). In CCR2 depleter mice, diphtheria toxoid (DT) administration results in $>99 \%$ depletion of bone marrow and tissue monocytes within 12 hours of injection ${ }^{17}$. To segregate any potential heterogeneity in CCR2 expression on non-myeloid cells we utilized adoptive transfer of melanoma antigen-specific CD8 T cells, which lack DTR transgene expression and have functional CCR2 for these experiments. Splenocytes from pmel-1 T cell receptor transgenic mice were treated in vitro with $\alpha-\mathrm{CD} 3 / \alpha-\mathrm{CD} 28$ and IL-2 to obtain a uniformly activated CD8+ T cell population specific for the melanosomal antigen gp100. After 3 days, CD8+ T cells were magnetically sorted from these cultures and transferred into B16-GM tumor bearing mice that had been reconstituted with CCR2-DTR Tg positive or negative bone marrow. All mice were treated with DT as indicated in the experimental schema (fig S3). DT treatment resulted in near complete elimination of CD11b+Ly6C+ (monocytic) cells and partial ablation of CD11b+Ly6C lo cells (macrophages) with preservation of $\mathrm{CD} 11 \mathrm{~b}+\mathrm{Ly} 6 \mathrm{G}+\mathrm{Ly} 6 \mathrm{C}$ intermediate (granulocytic) cells at the tumor site (fig 6A, and fig S3). Depletion of CCR2+ cells resulted in significantly greater accumulation of pmel $\mathrm{T}$ cells at the tumor site with a trend toward diminished $\mathrm{T}$ cells seen in the draining lymph node (fig 6B and 6C). The increased antigen specific $\mathrm{T}$ cell accumulation in the tumor resulting from the depletion of CCR2+ cells at this site resulted in significant delay in tumor growth in a parallel experiment (fig 6D). Depletion of CCR2+ cells alone lead to a growth delay, but importantly the combined effect was greatest with the highest pmel cell dose, underscoring the active role of antigen specific immunity in mediating an anti-tumor effect (fig 6E).

\section{CCR2 is expressed on melanoma patient MDSC}

We next sought to examine if CCR2 expression could characterize human MDSC. MDSC in patients with melanoma have been characterized by the expression of CD14 and low/absent expression of HLA-DR ${ }^{28}$. We find cells with this phenotypic description in greater frequency in the peripheral blood of patients with stage III and IV melanoma than in healthy donor controls and CCR2 is expression is seen in both groups. (fig. 7A and 7B). However, proliferation of CD8 $\mathrm{T}$ cells is enhanced in the absence of CCR2 expressing cells only in PBMC samples taken from patients with melanoma and not in healthy donor controls (fig. 7C). This strongly suggests that CCR2 expression characterizes cells with MDSC function in patients with melanoma. 


\section{Discussion}

There has been a great deal of interest in the immunostimulatory properties of granulocytemacrophage colony stimulating factor (GM-CSF) in autoimmune diseases and for immunotherapy in cancer ${ }^{29,30}$. More than 15 years ago, Dranoff and colleagues demonstrated that GM-CSF, in the context of $\gamma$-irradiated tumor cells, elicits potent immune responses in a murine model of melanoma ${ }^{22}$. This prompted the study of GM-CSF as an adjuvant to whole tumor, DNA, and peptide vaccination with promising results in a number of animal tumor models ${ }^{23,31-34}$. Similar strategies were safely performed in early phase clinical trials and immune responses were elicited ${ }^{35-38}$. However, in more recent randomized clinical trials, GM-CSF was found to have found detrimental effects on both immune responses and clinical outcomes ${ }^{5-7}$, a finding that may be related to the expansion of MDSC. Several groups have observed that GM-CSF dose and duration of exposure may mediate MDSC expansion 16,28,39-42. However, a direct connection between MDSC expansion and the failings of GM-CSF as a vaccine adjuvant has not been established.

To date, a specific characterization of GM-CSF expanded MDSC or a direct examination of MDSC effects on tumor immunity in vivo has been hampered by a lack of reagents to specifically target this population. Using a transgenic mouse with a CCR2 promoter driven GFP reporter, we demonstrate that chronic GM-CSF exposure expands and activates monocyte derived MDSC that express CCR2 and are direct descendents of CCR2expressing cells. Monocytic CCR2-expressing MDSC are present in multiple tumor types and in patients with melanoma(Fig 3C, S1, 7), suggesting that CCR2 is a generally applicable marker of this cell population. Additional studies are warranted to define the precise contribution of GM-CSF to MDSC function in tumor-bearing hosts as well as other pathologic conditions associated with MDSC accumulation.

In our depletion studies, we found that bone marrow-derived CCR2+ cells promote tumor growth and diminish the therapeutic effect of adoptively transferred activated antigenspecific $\mathrm{CD} 8+\mathrm{T}$ cells by preventing their infiltration into the tumor site (fig 6). Our studies utilized adoptively transferred activated antigen specific CD8+ T cells, a clinically valid approach, to examine the effects of CCR $2+$ cell depletion ${ }^{43}$. The use of adoptively transferred DTR transgene negative T cells excludes the possible effects of DT administration on endogenous CD8 T cells and permits the conclusion that CD8 T cell accumulation at the tumor site is inhibited by CCR $2+$ MDSC $^{26}$.

Although CCR2 enables the characterization of an MDSC subtype it is also found on a subset of CD4 and CD8 T cells ${ }^{26}$. In addition, in both naïve mice and B16 tumor bearing mice neither CCR2 reporter expression nor an MDSC population defined by other markers (CD11b/GR1, CD11b/F4/80, or CD11b/CD115) identifies a functional MDSC subtype ( ${ }^{39}$ and data not shown) presumably because the signals required to activate the suppressive program are not present under these conditions. Furthermore, our experiments in CCR2-I -mice demonstrate that MDSC retain functionality suggesting that signaling through this receptor is not required for suppressive function (Fig 5). Thus, CCR2 expression, but not signaling on MDSC could be used for targeting cell lineage specific reagents aimed at manipulating MDSC function in vivo.

It has been shown that CCR2 is important for MDSC trafficking to the tumor site ${ }^{25}$. We have also observed that accumulation of CCR2+MDSC at the tumor site is significantly diminished in CCR $2^{-1-}$ mice. Our numeric data suggests this is because these cells are less efficient at exiting the bone marrow space. A role for CCR2 in MDSC trafficking from the blood and lymphoid organs into tumors is not supported by our findings. CCR2 is required for monocyte egress from the bone marrow in the setting of acute inflammation, but other 
pathways may also play a role in the more chronic setting of tumor growth explaining the residual MDSC accumulation observed in B16-GM tumors ${ }^{24,25}$. It has been shown that the spleen can serve as a reservoir for monocytes that can be released in stress conditions in response to angiotensin $\mathrm{II}^{44}$. We did not observe further decreases in MDSC quantity in splenectomized CCR2-/- mice challenged with B16-GM tumors (data not shown) thus not supporting the notion that residual MDSC at the tumor site in CCR2-/- mice are released from the spleen. Additional studies are needed to define the full complement of pathways used by CCR2+MDSC for trafficking to the tumor site.

In summary, we have found that CCR 2 expression defines a monocyte-derived population of suppressive myeloid cells that regulates activated CD8 T cell entry into the tumor site. Our results suggest that combined targeting of CCR2+ MDSC as part of an immune based treatment strategy may improve the outcome of immunotherapy in the tumor-bearing host.

\section{Supplementary Material}

Refer to Web version on PubMed Central for supplementary material.

\section{Acknowledgments}

The authors wish to thank Andre Burey and Hong Zhong for providing technical support.

This work was supported by National Institute of Health grant KO8 AI071998 (to T. M. H), National Cancer Institute grants R01 CA56821, P01 CA33049, and P01 CA59350 (to J.DW. and A.N.H.), grant MRSG-11-054-01LIB from the American Cancer Society (to A.M.L.), grants from the Melanoma Research Alliance (to J.D.W. and A.M.L), The Lymphoma Foundation (to A.M.L. and A.N.H.), Medical Scientist Training Program grant GM07739 (to G.A.R)and grants from Swim Across America, the Lita Annenberg Hazen Foundation, the T.J. Martell Foundation, and the Mr. William H. Goodwin and Mrs. Alice Goodwin and the Commonwealth Cancer Foundation for Research (to J.D.W. and A.N.H.).

\section{References}

1. Turk MJ, Guevara-Patino JA, Rizzuto GA, Engelhorn ME, Sakaguchi S, Houghton AN. Concomitant tumor immunity to a poorly immunogenic melanoma is prevented by regulatory $\mathrm{T}$ cells. J Exp Med. 2004; 200:771-782. [PubMed: 15381730]

2. Quezada SA, Peggs KS, Simpson TR, Shen Y, Littman DR, Allison JP. Limited tumor infiltration by activated $\mathrm{T}$ effector cells restricts the therapeutic activity of regulatory $\mathrm{T}$ cell depletion against established melanoma. J Exp Med. 2008; 205:2125-2138. [PubMed: 18725522]

3. Peranzoni E, Zilio S, Marigo I, et al. Myeloid-derived suppressor cell heterogeneity and subset definition. Curr Opin Immunol. 2010; 22:238-244. [PubMed: 20171075]

4. Gabrilovich DI, Nagaraj S. Myeloid-derived suppressor cells as regulators of the immune system. Nat Rev Immunol. 2009; 9:162-174. [PubMed: 19197294]

5. Small, E.; Demkow, T.; Gerritsen, WR., et al. A phase III trial of GVAX immunotherapy for prostate cancer in combination with docetaxel vs. docetaxel plus prednisone in symptomatic, castration-resistant prostate cancer (CRPC). ASCO GU; 2009. p. Abs 7

6. Faries MB, Hsueh EC, Ye X, Hoban M, Morton DL. Effect of granulocyte/macrophage colonystimulating factor on vaccination with an allogeneic whole-cell melanoma vaccine. Clin Cancer Res. 2009; 15:7029-7035. [PubMed: 19903777]

7. Slingluff CL Jr, Petroni GR, Olson WC, et al. Effect of granulocyte/macrophage colony-stimulating factor on circulating CD8+ and CD4+ T-cell responses to a multipeptide melanoma vaccine: outcome of a multicenter randomized trial. Clin Cancer Res. 2009; 15:7036-7044. [PubMed: 19903780]

8. Sica A, Bronte V. Altered macrophage differentiation and immune dysfunction in tumor development. J Clin Invest. 2007; 117:1155-1166. [PubMed: 17476345]

9. Mandruzzato S, Solito S, Falisi E, et al. IL4Ralpha+ myeloid-derived suppressor cell expansion in cancer patients. J Immunol. 2009; 182:6562-6568. [PubMed: 19414811] 
10. Youn JI, Gabrilovich DI. The biology of myeloid-derived suppressor cells: the blessing and the curse of morphological and functional heterogeneity. Eur J Immunol. 40:2969-2975. [PubMed: 21061430]

11. Huang B, Pan PY, Li Q, et al. Gr-1+CD115+ immature myeloid suppressor cells mediate the development of tumor-induced $\mathrm{T}$ regulatory cells and T-cell anergy in tumor-bearing host. Cancer Res. 2006; 66:1123-1131. [PubMed: 16424049]

12. Gallina G, Dolcetti L, Serafini P, et al. Tumors induce a subset of inflammatory monocytes with immunosuppressive activity on CD8+ T cells. 2006; 116:2777-2790.

13. Kusmartsev S, Gabrilovich DI. STAT1 Signaling Regulates Tumor-Associated MacrophageMediated T Cell Deletion. 2005; 174:4880-4891.

14. Movahedi K, Guilliams M, Van den Bossche J, et al. Identification of discrete tumor-induced myeloid-derived suppressor cell subpopulations with distinct $\mathrm{T}$ cell-suppressive activity. Blood. 2008; 111:4233-4244. [PubMed: 18272812]

15. Youn JI, Nagaraj S, Collazo M, Gabrilovich DI. Subsets of myeloid-derived suppressor cells in tumor-bearing mice. J Immunol. 2008; 181:5791-5802. [PubMed: 18832739]

16. Dolcetti L, Peranzoni E, Ugel S, et al. Hierarchy of immunosuppressive strength among myeloidderived suppressor cell subsets is determined by GM-CSF. Eur J Immunol. 2010; 40:22-35. [PubMed: 19941314]

17. Hohl TM, Rivera A, Lipuma L, et al. Inflammatory monocytes facilitate adaptive CD4 T cell responses during respiratory fungal infection. Cell Host Microbe. 2009; 6:470-481. [PubMed: 19917501]

18. Overwijk WW, Theoret MR, Finkelstein SE, et al. Tumor regression and autoimmunity after reversal of a functionally tolerant state of self-reactive CD8+ T cells. J Exp Med. 2003; 198:569_ 580. [PubMed: 12925674]

19. Hara I, Nguyen H, Takechi Y, Gansbacher B, Chapman PB, Houghton AN. Rejection of mouse melanoma elicited by local secretion of interleukin-2: implicating macrophages without $\mathrm{T}$ cells or natural killer cells in tumor rejection. Int J Cancer. 1995; 61:253-260. [PubMed: 7705956]

20. Hara I, Takechi Y, Houghton AN. Implicating a role for immune recognition of self in tumor rejection: passive immunization against the brown locus protein. J Exp Med. 1995; 182:16091614. [PubMed: 7595233]

21. Foster BA, Gingrich JR, Kwon ED, Madias C, Greenberg NM. Characterization of prostatic epithelial cell lines derived from transgenic adenocarcinoma of the mouse prostate (TRAMP) model. Cancer Res. 1997; 57:3325-3330. [PubMed: 9269988]

22. Dranoff G, Jaffee E, Lazenby A, et al. Vaccination with irradiated tumor cells engineered to secrete murine granulocyte-macrophage colony-stimulating factor stimulates potent, specific, and long-lasting anti-tumor immunity. Proc Natl Acad Sci U S A. 1993; 90:3539-3543. [PubMed: 8097319]

23. Chang DZ, Lomazow W, Joy Somberg C, Stan R, Perales MA. Granulocyte-macrophage colony stimulating factor: an adjuvant for cancer vaccines. Hematology. 2004; 9:207-215. [PubMed: 15204102]

24. Serbina NV, Pamer EG. Monocyte emigration from bone marrow during bacterial infection requires signals mediated by chemokine receptor CCR2. Nat Immunol. 2006; 7:311-317. [PubMed: 16462739]

25. Sawanobori Y, Ueha S, Kurachi M, et al. Chemokine-mediated rapid turnover of myeloid-derived suppressor cells in tumor-bearing mice. Blood. 2008; 111:5457-5466. [PubMed: 18375791]

26. Mack M, Cihak J, Simonis C, et al. Expression and characterization of the chemokine receptors CCR2 and CCR5 in mice. J Immunol. 2001; 166:4697-4704. [PubMed: 11254730]

27. Saio M, Radoja S, Marino M, Frey AB. Tumor-infiltrating macrophages induce apoptosis in activated CD8(+) T cells by a mechanism requiring cell contact and mediated by both the cellassociated form of TNF and nitric oxide. J Immunol. 2001; 167:5583-5593. [PubMed: 11698429]

28. Filipazzi P, Valenti R, Huber V, et al. Identification of a new subset of myeloid suppressor cells in peripheral blood of melanoma patients with modulation by a granulocyte-macrophage colonystimulation factor-based antitumor vaccine. J Clin Oncol. 2007; 25:2546-2553. [PubMed: 17577033] 
29. Hamilton JA. Colony-stimulating factors in inflammation and autoimmunity. Nat Rev Immunol. 2008; 8:533-544. [PubMed: 18551128]

30. Le DT, Pardoll DM, Jaffee EM. Cellular vaccine approaches. Cancer J. 2010; 16:304-310. [PubMed: 20693840]

31. Perales MA, Fantuzzi G, Goldberg SM, et al. GM-CSF DNA induces specific patterns of cytokines and chemokines in the skin: implications for DNA vaccines. Cytokines Cell Mol Ther. 2002; 7:125-133. [PubMed: 12850812]

32. Bergman PJ, Camps-Palau MA, McKnight JA, et al. Development of a xenogeneic DNA vaccine program for canine malignant melanoma at the Animal Medical Center. Vaccine. 2006; 24:45824585. [PubMed: 16188351]

33. Simmons AD, Li B, Gonzalez-Edick M, et al. GM-CSF-secreting cancer immunotherapies: preclinical analysis of the mechanism of action. Cancer Immunol Immunother. 2007; 56:16531665. [PubMed: 17410360]

34. Ferrone CR, Perales MA, Goldberg SM, et al. Adjuvanticity of plasmid DNA encoding cytokines fused to immunoglobulin Fc domains. Clin Cancer Res. 2006; 12:5511-5519. [PubMed: 17000687]

35. Atzpodien J, Reitz M. GM-CSF plus antigenic peptide vaccination in locally advanced melanoma patients. Cancer Biother Radiopharm. 2007; 22:551-555. [PubMed: 17803450]

36. Perales MA, Yuan J, Powel S, et al. Phase I/II Study of GM-CSF DNA as an Adjuvant for a Multipeptide Cancer Vaccine in Patients With Advanced Melanoma. Mol Ther. 2008

37. Bins A, Mallo H, Sein J, et al. Phase I clinical study with multiple peptide vaccines in combination with tetanus toxoid and GM-CSF in advanced-stage HLA-A*0201-positive melanoma patients. J Immunother. 2007; 30:234-239. [PubMed: 17471170]

38. Cassaday RD, Sondel PM, King DM, et al. A phase I study of immunization using particlemediated epidermal delivery of genes for gp100 and GM-CSF into uninvolved skin of melanoma patients. Clin Cancer Res. 2007; 13:540-549. [PubMed: 17255276]

39. Bronte V, Chappell DB, Apolloni E, et al. Unopposed Production of Granulocyte-Macrophage Colony-Stimulating Factor by Tumors Inhibits CD8+ T Cell Responses by Dysregulating AntigenPresenting Cell Maturation. 1999; 162:5728-5737.

40. Serafini P, Carbley R, Noonan KA, Tan G, Bronte V, Borrello I. High-dose granulocytemacrophage colony-stimulating factor-producing vaccines impair the immune response through the recruitment of myeloid suppressor cells. Cancer Res. 2004; 64:6337-6343. [PubMed: 15342423]

41. Marigo I, Bosio E, Solito S, et al. Tumor-induced tolerance and immune suppression depend on the C/EBPbeta transcription factor. Immunity. 2010; 32:790-802. [PubMed: 20605485]

42. Parmiani G, Castelli C, Pilla L, Santinami M, Colombo MP, Rivoltini L. Opposite immune functions of GM-CSF administered as vaccine adjuvant in cancer patients. Ann Oncol. 2007; 18:226-232. [PubMed: 17116643]

43. Rosenberg SA, Restifo NP, Yang JC, Morgan RA, Dudley ME. Adoptive cell transfer: a clinical path to effective cancer immunotherapy. Nat Rev Cancer. 2008; 8:299-308. [PubMed: 18354418]

44. Leuschner F, Panizzi P, Chico-Calero I, et al. Angiotensin-converting enzyme inhibition prevents the release of monocytes from their splenic reservoir in mice with myocardial infarction. Circ Res. 2010; 107:1364-1373. [PubMed: 20930148] 
A

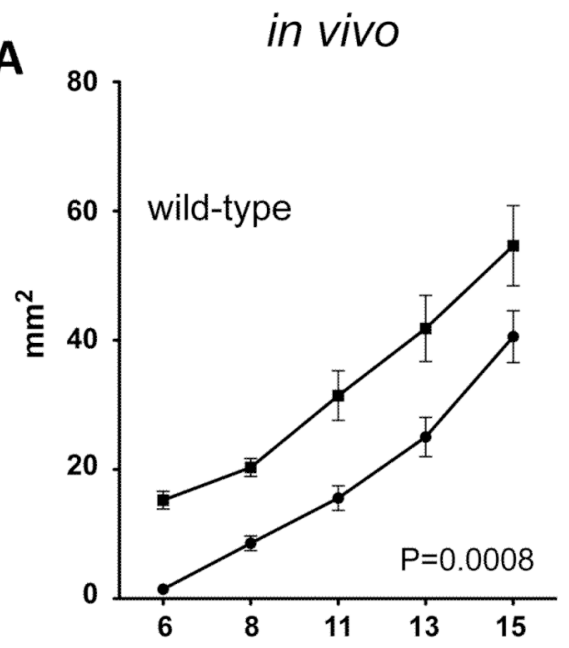

C

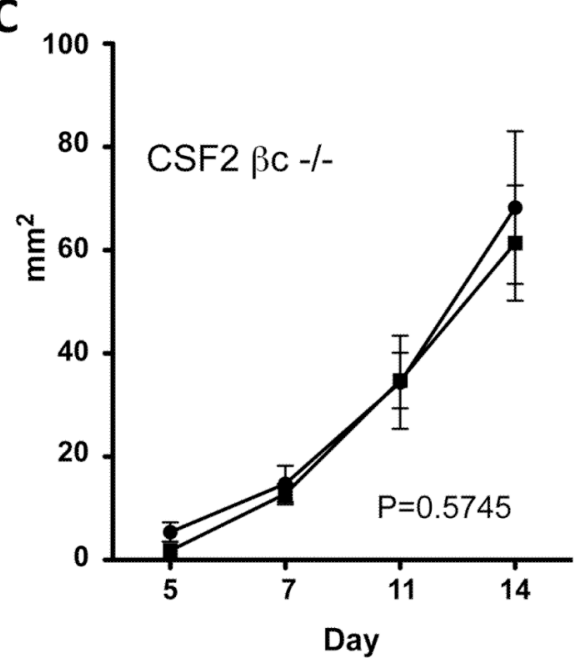

B

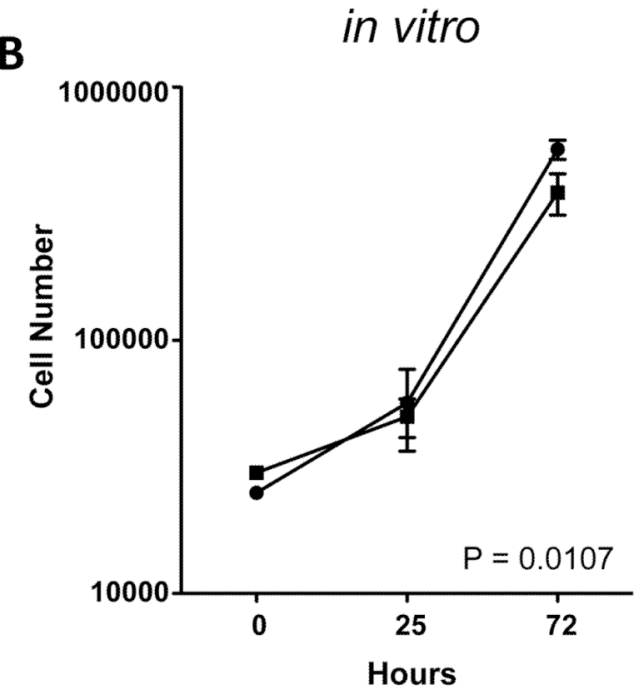

- B16 - B16-GM

Figure 1. Chronic GM-CSF promotes tumor growth in vivo

Mice were inoculated intradermally with $1.25 \times 10^{5}$ B16 or B16-GM tumor cells. Growth was measured in 2 perpendicular dimensions every 2-3 days. Average tumor size is depicted at each timepoint. C57B16/J mice ( $\mathrm{n}=10$ /group) (A); CSF2R $\beta \mathrm{c}-/-$ mice ( $\mathrm{n}=10 /$ group) (C). B16 or B16-GM cells were plated in 6-well plates in triplicate. Cells were harvested and counted at the indicated time interval (B). Representative experiments of 2-3 replicates are shown 
A
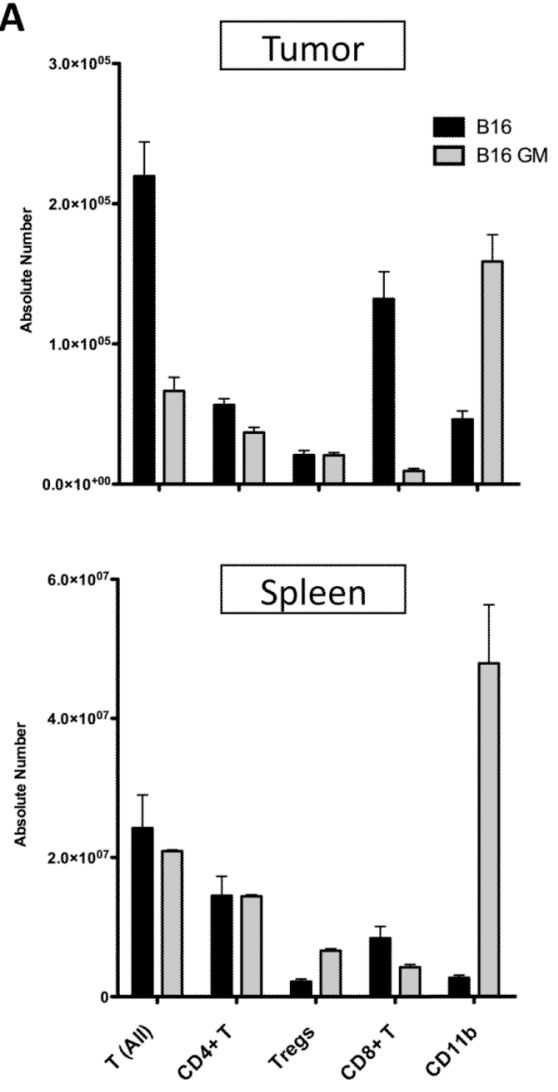

B
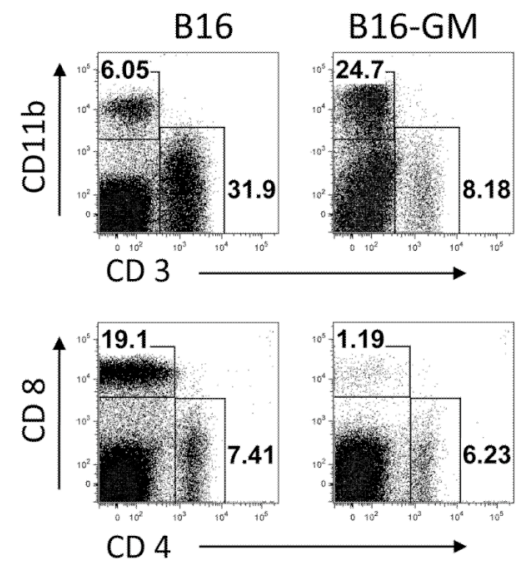

C

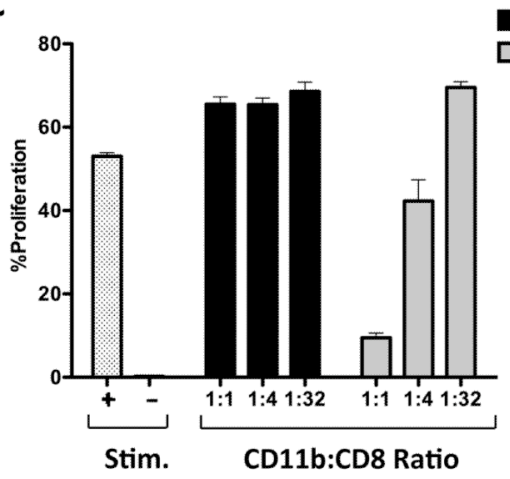

Figure 2. Tumor derived GM-CSF recruits an immune inhibitory cellular infiltrate dominated by myeloid derived suppressor cells

C57BL/6 mice were inoculated with $5 \times 10^{5} \mathrm{~B} 16$ or B16GM cells in matrigel. Tumors were harvested 13 days later, stained for surface markers and analyzed by flow cytometry. $(n=3 /$ tumor type). Results for T cells (CD3+), CD4+ T (CD4+/CD3+), CD8+ T (CD8+/CD3+), Tregs (CD4+/FoxP3+), and CD11b cells (A); Representative plots (B). Suppression assay: $\mathrm{CD} 11 \mathrm{~b}+$ cells were flow sorted from B16 or B16-GM tumors as described in the methods and co-cultured with naïve CFSE-labeled CD $8+\mathrm{T}$ cells plated onto $\alpha-\mathrm{CD} 3 / \alpha-\mathrm{CD} 28$ coated 96-well plates in the ratios indicated. Stim + are wells without CD11b+ cells and Stim - are wells without MDSC or antibody. \%CD8+ T cell proliferation was measured by assessing CFSE dilution by flow cytometry after 72 hours of culture (C). Representative examples of 2-3 replicate experiments are shown. 
A

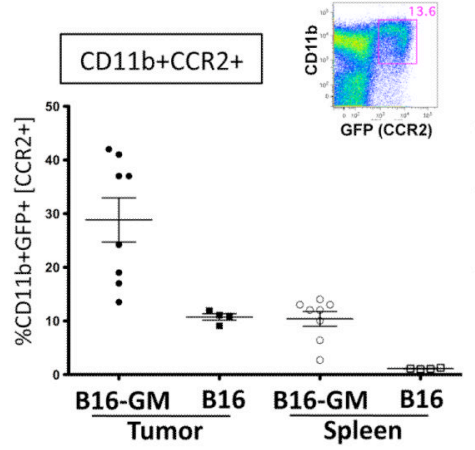

C

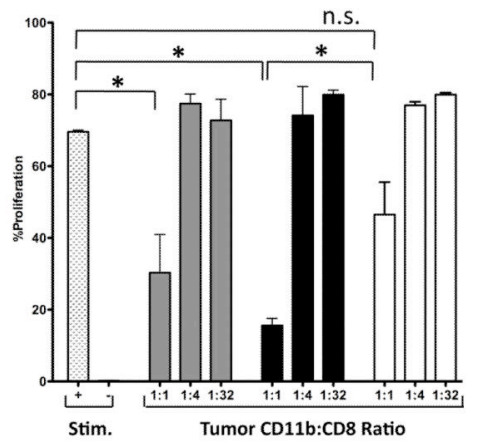

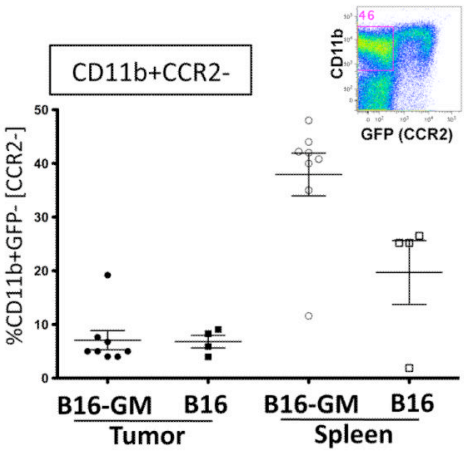
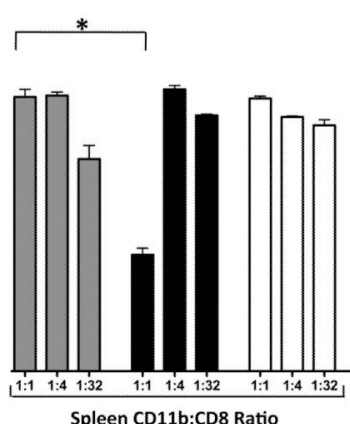

B
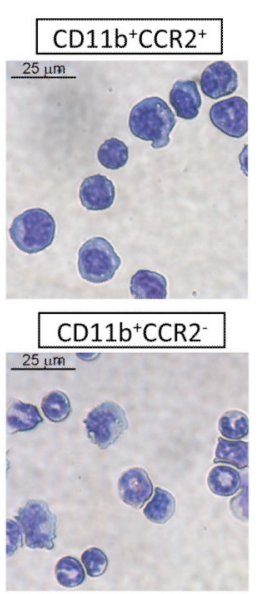

Figure 3. CCR2 reporter expression segregates a monocytic CD11b+ myeloid derived suppressor cell in the tumor-bearing host CCR2 reporter mice (4-8/group) were inoculated with B16-GM or B16 tumor cells in Matrigel $^{\text {TM }}$. Tumors and spleens were harvested after 2 weeks or when tumors were of equivalent size (500-800mg) and analyzed for GFP expression in CD45+CD11b+ cells by flow cytometry. The quantity of $\mathrm{CD} 11 \mathrm{~b}+\mathrm{GFP}+(\mathrm{CCR} 2+)$ and $\mathrm{GFP}-(\mathrm{CCR} 2-)$ cells as a percentage of the immune infiltrate $(\% \mathrm{CD} 45+$ cells) is shown. One representative spleen sample is depicted to show each population. Data is composite of 2 separate experiments (A). In separate experiments cytospins of flow-sorted cells were stained with a Diff-Quick stain set (Dade Behring, inc.) and evaluated at 40X magnification (B). Single cell suspensions harvested from tumors and spleens 2 weeks after tumor inoculation were flowsorted for $\mathrm{CD} 11 \mathrm{~b}+\mathrm{CD} 45+$ cells and then additionally sorted for CCR2+ or - populations based on GFP reporter expression. Each of the three subsets was individually tested for its ability to suppress the proliferation of CD8+ T cells stimulated with $\alpha-\mathrm{CD} 3 / \alpha-\mathrm{CD} 28$ antibody (Stim). Stim + are wells without CD11b+ cells and Stim - are wells without MDSC or antibody. Proliferation was measured after 72 hours of co-culture with the indicated ratio of MDSC (C). 

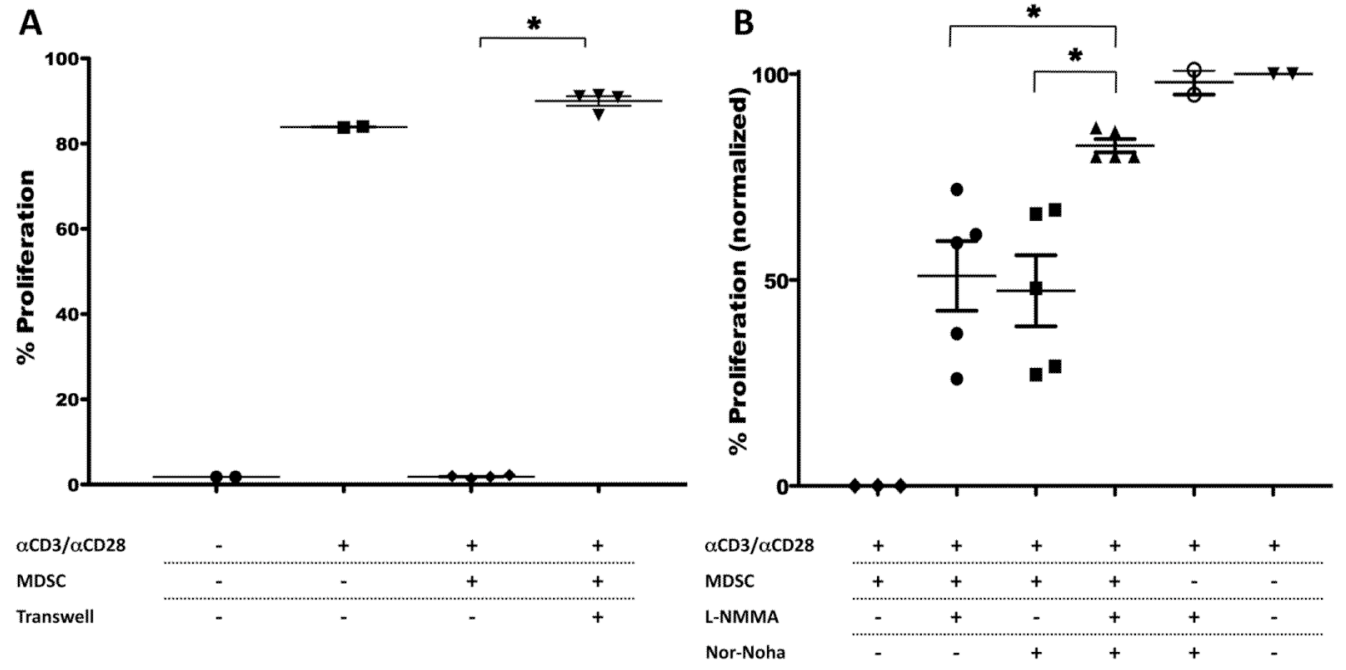

Figure 4. GM-CSF expands and activates monocytic CCR2+ CD11b+ MDSC that suppress CD8+ $T$ cell proliferation via the coordinated action of iNOS and arginase in a contact dependent fashion

CCR2-GFP mice (3-6/group) were subcutaneously inoculated with B16-GM embedded in Matrigel $^{\mathrm{TM}}$. Single cell suspensions harvested from spleens 2 weeks later were flow-sorted for CD11b+CD45+ CCR2 reporter + cells (MDSC), which were added to CD8+ T cells stimulated with $\alpha-C D 3 / \alpha-C D 28$ antibody. Proliferation was measured after 72 hours of coculture in the indicated conditions (A). CD8+ T cell suppressive capability measured with MDSC in contact with CD8+ $\mathrm{T}$ cells or placed inside a transwell $(0.4 \mu \mathrm{M})$ insert (A). CD8+ $\mathrm{T}$ cell proliferation in the presence of inhibitors of iNOS (L-NMMA) and/or arginase (NorNOHA); proliferation is normalized across assays to the baseline proliferation in the absence of CCR2+CD11b+ MDSC (B). One representative experiment of 2 similar replicates is shown in $\mathrm{A}$. In $\mathrm{B}$ combined results from 3 individual assays are shown. * $\mathrm{P}<0.05$ 

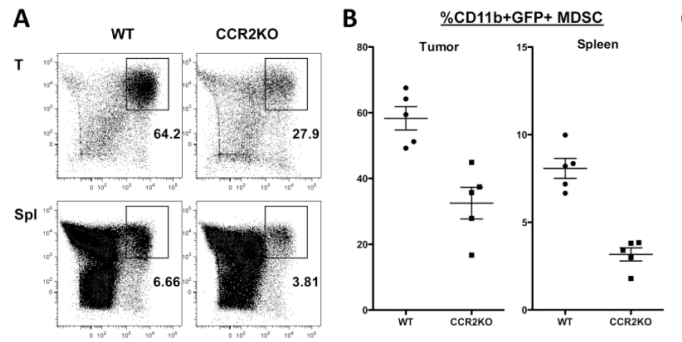

C $\square$ WT $\square$ CСR2KO
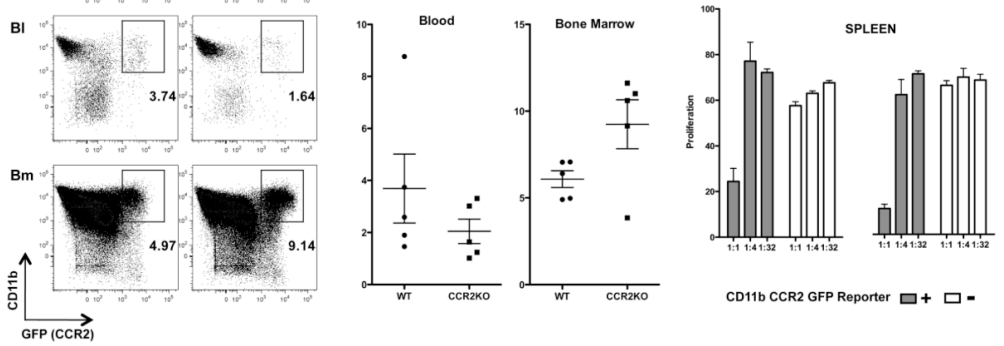

Figure 5. CCR2 signaling is not required for MDSC function

CCR2-GFP transgene positive mice on a CCR2-wt or CCR2-/- background were inoculated with B16-GM tumors in Matrigel $^{\mathrm{TM}}$. Tissues were harvested on day 11 after inoculation and CD11b+GFP+ cells enumerated by flow cytometry. Representative flow cytometry plots (A). Quantitative data ( $n=5$ /group) showing quantity of CD11b+GFP+ cells expressed as a percent of single, live, CD45+ cells(B). CD45+CD11b+GFP+ cells were sorted from tumor or spleen of animals challenged with B16-GM tumors 14 days prior and added to CD8 T cells stimulated with $\alpha-C D 3 / \alpha-C D 28$ antibody. Proliferation was measured after 72 hours of co-culture with the indicated ratio of $\operatorname{MDSC}(\mathrm{C})$. One of $2(\mathrm{~A}, \mathrm{~B})$ or $3(\mathrm{C})$ representative experiments is shown. 
A

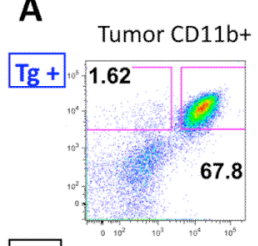

$\mathrm{Tg}-$

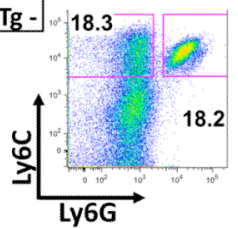

B

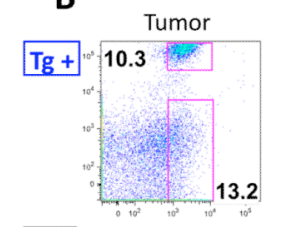

$\mathrm{Tg}-0.056$

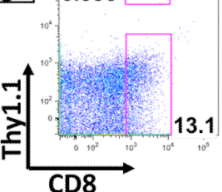

CD8

D

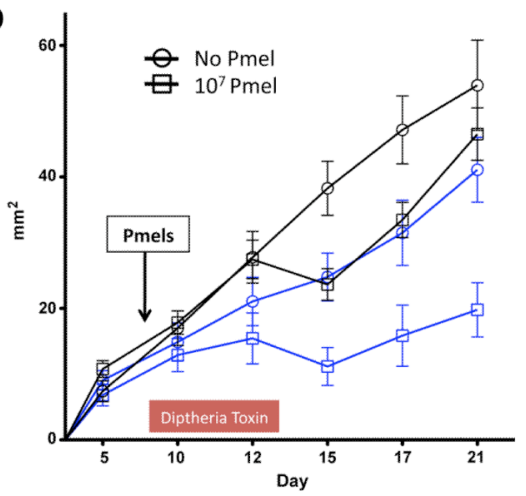

C
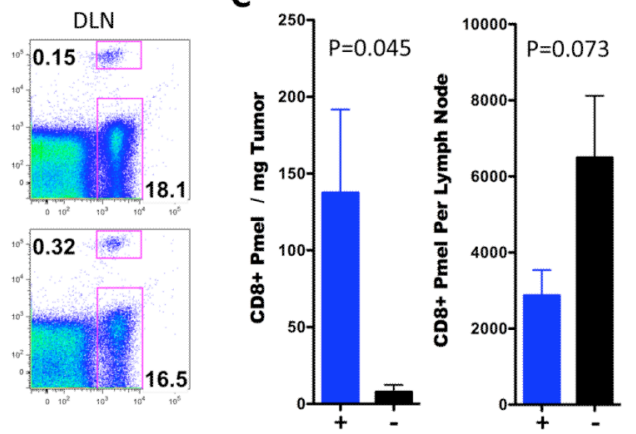

$E$

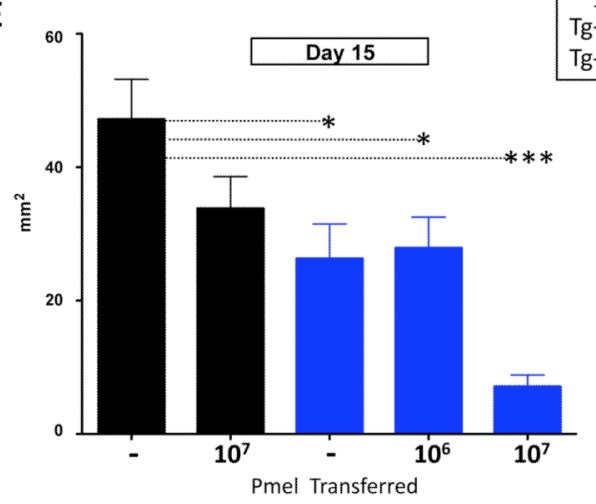

Figure 6. Depletion of CCR2+ cells increases antigen specific $T$ cells in the tumor microenvironment and results in diminished tumor growth

CD8+ pmel cells were magnetically sorted for adoptive transfer experiments from spleen cells previously stimulated in vitro with soluble $\alpha$-CD3 $(1 \mu \mathrm{g} / \mathrm{ml}) / \alpha$-CD28 $(2 \mu \mathrm{g} / \mathrm{ml})$ antibody for 72 hours and IL-2 (30 million units $/ \mathrm{ml}$ ) for 24 hours. Diphtheria toxin receptor transgene (CCR2-DTR Tg) positive and transgene negative $(\mathrm{Tg}-)$ mice were inoculated with $1.25 \times 10^{5}$ B16-GM i.d. and treated with daily injections of diphtheria toxoid $250 \mathrm{ng}$ intraperitoneally on day $8-13$. Monocytic and granulocytic myeloid cell subsets in the tumor microenvironment of $\mathrm{Tg}+$ and $\mathrm{Tg}-$ mice after treatment (A). Adoptively transferred $\mathrm{T}$ cell accumulation at the tumor site of $\mathrm{Tg}+$ and $\mathrm{Tg}-$ mice 6 days after the transfer of $6 \times 10^{5}$ activated CD8+ pmel T cells in representative mice (B) and average in each group of 5 mice at the tumor site and draining lymph node (C). Tumor growth in mice ( $\mathrm{n}=10-12 /$ group) treated with increasing doses of CD8+ pmel T cell transfer and DT (D). Individual effect of administration of DT versus increasing doses of CD8 pmels; *p<0.05, *** $\mathrm{p}<0.0001$ (E). One representative example is shown of 2-3 similar experiments for each figure. 
A

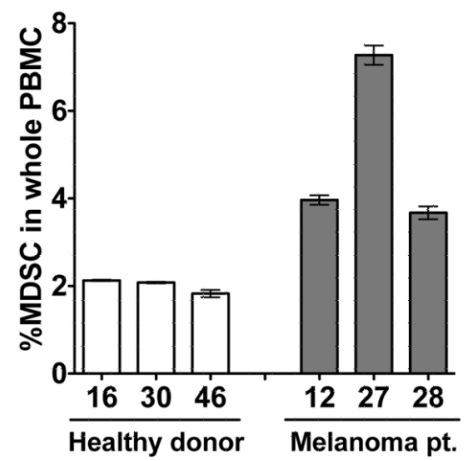

B
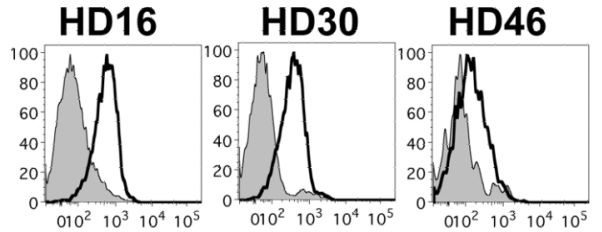

Pt.12

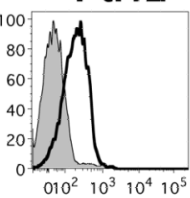

Pt.27

Pt.28
C
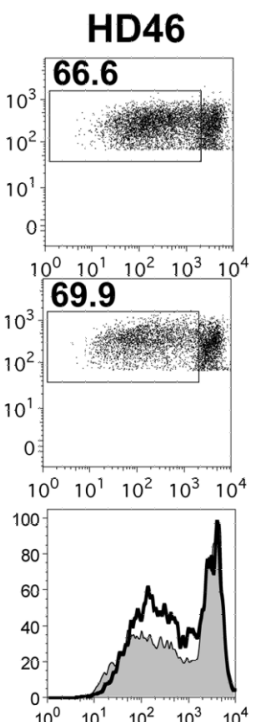

Pt.27

68.5
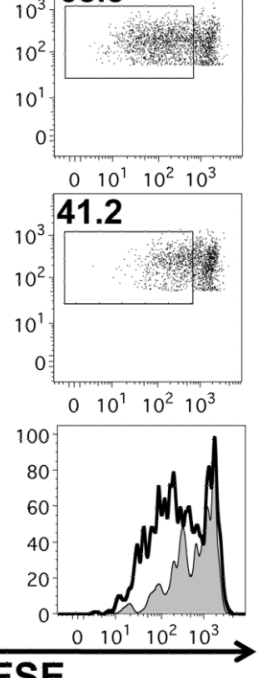

$\underline{\text { CCR2+ Cells }}$

Absent

Present

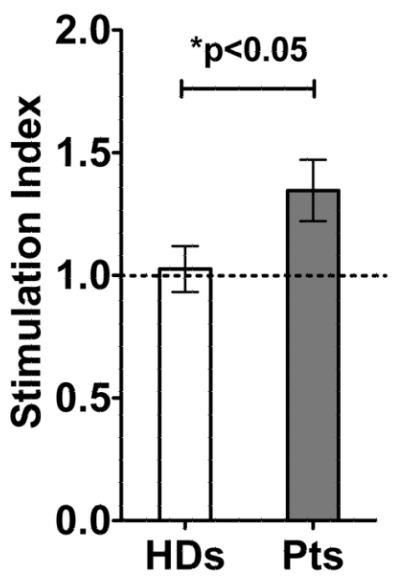

Figure 7. CCR2 expression on melanoma patient MDSC

Healthy donor(HD) or stage III and IV melanoma patient(Pt) $\mathrm{PBMC}$ were evaluated by flow cytometry for the quantity of lineage negative (CD3, CD16, CD19, CD20, CD56), CD14+, HLA-DR low/neg cells (A). The expression of CCR2 was measured in comparison with isotype control in each subject $(\mathrm{n}=3 \mathrm{HD}, 3 \mathrm{Pt})(\mathrm{B})$. PBMC depleted of CCR2 expressing cells were stimulated to proliferate with OKT-3 and IL-2. CFSE dilution of CD8 T cells in the culture is shown in the absence of CCR2+ cells or with CCR2+ cells added back. The average stimulation index is graphed for 3 healthy donor and 3 melanoma patient PBMC (C). Stimulation index $=\left(\%\right.$ proliferated $\mathrm{CD}^{+} \mathrm{T}$ cells in CCR 2 depleted $\left.\mathrm{PBMC}\right) \div(\%$ proliferated $\mathrm{CD}^{+} \mathrm{T}$ cell in CCR2-PBMC with $\mathrm{CCR} 2+$ cell added back). 